Swarthmore College

Works

$12-1-2001$

\title{
Field-Dependent Tilt And Birefringence Of Electroclinic Liquid Crystals: Theory And Experiment
}

\author{
J. V. Selinger \\ Peter J. Collings \\ Swarthmore College, pcollin1@swarthmore.edu \\ R. Shashidhar
}

Follow this and additional works at: https://works.swarthmore.edu/fac-physics

Part of the Physics Commons

Let us know how access to these works benefits you

\section{Recommended Citation}

J. V. Selinger, Peter J. Collings, and R. Shashidhar. (2001). "Field-Dependent Tilt And Birefringence Of Electroclinic Liquid Crystals: Theory And Experiment". Physical Review E. Volume 64, Issue 6. DOI: 10.1103/PhysRevE.64.061705

https://works.swarthmore.edu/fac-physics/155

This work is brought to you for free by Swarthmore College Libraries' Works. It has been accepted for inclusion in Physics \& Astronomy Faculty Works by an authorized administrator of Works. For more information, please contact myworks@swarthmore.edu. 


\title{
Field-dependent tilt and birefringence of electroclinic liquid crystals: Theory and experiment
}

\author{
Jonathan V. Selinger, ${ }^{1}$ Peter J. Collings, ${ }^{1,2}$ and R. Shashidhar ${ }^{1}$ \\ ${ }^{1}$ Center for Bio/Molecular Science and Engineering, Naval Research Laboratory, Code 6900, 4555 Overlook Avenue, SW, \\ Washington, D.C. 20375 \\ ${ }^{2}$ Department of Physics and Astronomy, Swarthmore College, Swarthmore, Pennsylvania 19081
}

(Received 30 July 2001; published 27 November 2001)

\begin{abstract}
An unresolved issue in the theory of liquid crystals is the molecular basis of the electroclinic effect in the smectic- $A$ phase. Recent $x$-ray scattering experiments suggest that, in a class of siloxane-containing liquid crystals, an electric field changes a state of disordered molecular tilt in random directions into a state of ordered tilt in one direction. To investigate this issue, we measure the optical tilt and birefringence of these liquid crystals as functions of field and temperature, and we develop a theory for the distribution of molecular orientations under a field. A comparison of theory and experiment confirms that these materials have a disordered distribution of molecular tilt directions that is aligned by an electric field, giving a large electroclinic effect. It also shows that the effective dipole moment, a key parameter in the theory, scales as a power law near the smectic- $A$-smectic- $C$ transition.
\end{abstract}

DOI: 10.1103/PhysRevE.64.061705

PACS number(s): 61.30.Cz, 61.30.Gd, 64.70.Md

\section{INTRODUCTION}

In liquid crystals, an applied electric field influences the orientational order of the molecules. In particular, in the smectic- $A(\mathrm{Sm}-A)$ phase of chiral liquid crystals, an electric field applied in the smectic layer plane induces a molecular tilt relative to the layer normal. The magnitude of the tilt varies continuously with electric field, and the direction of the tilt is orthogonal to the field. This coupling between an electric field and the molecular tilt is called the electroclinic effect. It was predicted on the basis of symmetry [1] and was subsequently observed experimentally [2]. It is now being developed for use in electro-optic devices in which the continuously variable tilt leads to a gray scale [3-6].

Most theoretical understanding of the electroclinic effect has been developed through Landau theory, which minimizes the free energy expanded in powers of the molecular tilt and polarization $[1,7,8]$. This phenomenological approach explains macroscopic aspects of the electroclinic effect. It shows that the tilt depends linearly on electric field for low fields, and that the coefficient of the linear dependence diverges as the system approaches a second-order transition from the $\mathrm{Sm}-A$ to the smectic- $C$ ( $\mathrm{Sm}-C$ ) phase. However, the microscopic basis of the electroclinic effect is still unresolved. Key questions are: What is the distribution of molecular orientations, and how does this distribution change under an applied electric field?

There have been two general concepts about the microscopic basis of the electroclinic effect. In the first scenario, the molecules all stand perpendicular to the smectic layers in the absence of a field, and they reorient together as rigid rods under a field. In the second scenario, the molecules have a random distribution of azimuthal orientations about a tilt cone before the field is applied, and they become ordered in a single tilted direction under a field. The latter scenario is suggested by the de Vries description of the Sm- $A$ phase [9]. Each of these concepts is consistent with a net observed tilt that scales linearly with applied electric field for low fields, and then saturates at high fields. Thus, the issue is how to distinguish between these possibilities.

One way to distinguish between these microscopic scenarios is through molecular-scale simulations. Our group has carried out Monte Carlo simulations of Sm- $A$ liquid crystals under an applied electric field [10]. These simulations use a model molecular structure consisting of seven soft spheres bonded rigidly together in the biaxial zigzag shape of the letter Z. A transverse electric dipole moment makes the molecules chiral. These simulations show a strong electroclinic effect, which involves a combination of the "rigid-rod" and "de Vries" scenarios. In the absence of an electric field, the molecules have a distribution of orientations, with vortex defects in the smectic layers. When an electric field is applied, the magnitude of the tilt increases and the azimuthal orientation of the tilt becomes ordered, perpendicular to the electric field. Thus, the simulations show that both of these scenarios can occur in model liquid crystals. They do not, however, show which of these scenarios plays the dominant role in actual experimental materials.

To distinguish between these possibilities in experimental materials, several studies have examined the smectic layer spacing as a function of applied electric field. The two scenarios make very different predictions for the smectic layer spacing. In the rigid-rod scenario, when the molecules tilt by an angle $\theta$, the smectic layer spacing should contract by a factor of $\cos \theta$. By contrast, in the de Vries scenario, the molecules are already tilted in zero field, and a field only orders the azimuthal direction of the tilt, so the field should not induce any layer contraction. The experimental studies have found that most "conventional" $\mathrm{Sm}-A$ liquid crystals show a field-induced layer contraction, consistent with the prediction of the rigid-rod scenario. This contraction can be seen in measurements of the layer spacing through x-ray diffraction [11]. It can also be seen through field-induced layer buckling, which gives an optical stripe pattern [11-15]. However, certain materials have been developed that show a substantial electroclinic tilt with hardly any layer contrac- 


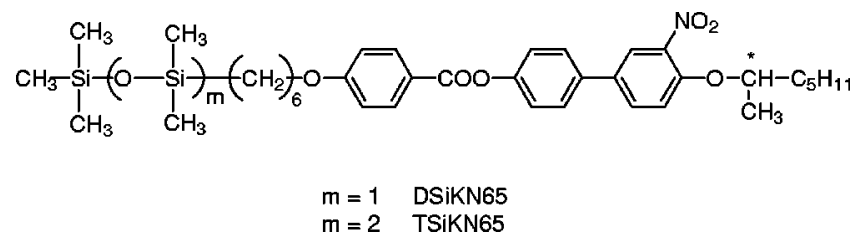

FIG. 1. Molecular structure of the siloxane-containing liquid crystals studied in this paper.

tion, consistent with the de Vries scenario. These include compounds with a fluoroether tail [16], a chiral lactic ester in the tail [17], and dimethylsiloxane groups in the tail [18]. In fact, an optical and x-ray study of one organosiloxane compound revealed tilt angles as large as $31^{\circ}$ in the $\mathrm{Sm}-A$ phase with a layer contraction of less than $1 \%$ [19].

The purpose of our current study is to explore a different way of distinguishing between these possibilities. Instead of measuring the smectic layer spacing, we investigate the optical birefringence as a function of applied electric field. The birefringence is the difference between indices of refraction for light that is linearly polarized parallel or perpendicular to the average director of a sample. It is an appropriate probe for the microscopic basis of the electroclinic effect because it is sensitive to the degree of orientational order. The rigid-rod and de Vries scenarios make different predictions for the birefringence as a function of electric field. In the rigid-rod scenario, the molecules have strong orientational order even in zero field, so the zero-field birefringence should be high. When an electric field is applied, the molecules remain parallel to each other in a tilted orientation, and hence the birefringence should vary only weakly as function of field [20]. By contrast, in the de Vries scenario, the molecules have a distribution of orientations about a tilt cone in zero field. The zero-field birefringence should be greatly reduced because of the orientational averaging about the tilt cone. When an electric field is applied, the molecules become more aligned with each other in a particular tilted orientation. As a result, the birefringence of a de Vries-type material should increase substantially with the applied field.

In a preliminary communication, our group reported experiments on the optical tilt and birefringence of four electroclinic liquid crystals with closely related chemical structures: KN125, SiKN105, DSiKN65, and TSiKN105 [21]. In these abbreviations, $\mathrm{KN}$ is a label, the numbers on the right refer to the length of the hydrocarbon chains, and the letters on the left refer to siloxane units in the latter three compounds. KN125 is believed to follow the rigid-rod scenario for the electroclinic effect (based on a substantial layer contraction and buckling [11]), while the three siloxanecontaining compounds are believed to follow the de Vries scenario (based on the lack of layer buckling). Our experiments confirmed that KN125 has a large and weakly fielddependent birefringence, while the siloxane-containing compounds have a much smaller and more strongly fielddependent birefringence. To analyze the data, we developed a model for the birefringence as a function of the field in de Vries-type materials, based on averaging the molecular dielectric tensor over a field-dependent orientational distribution function. This model was consistent with the observed
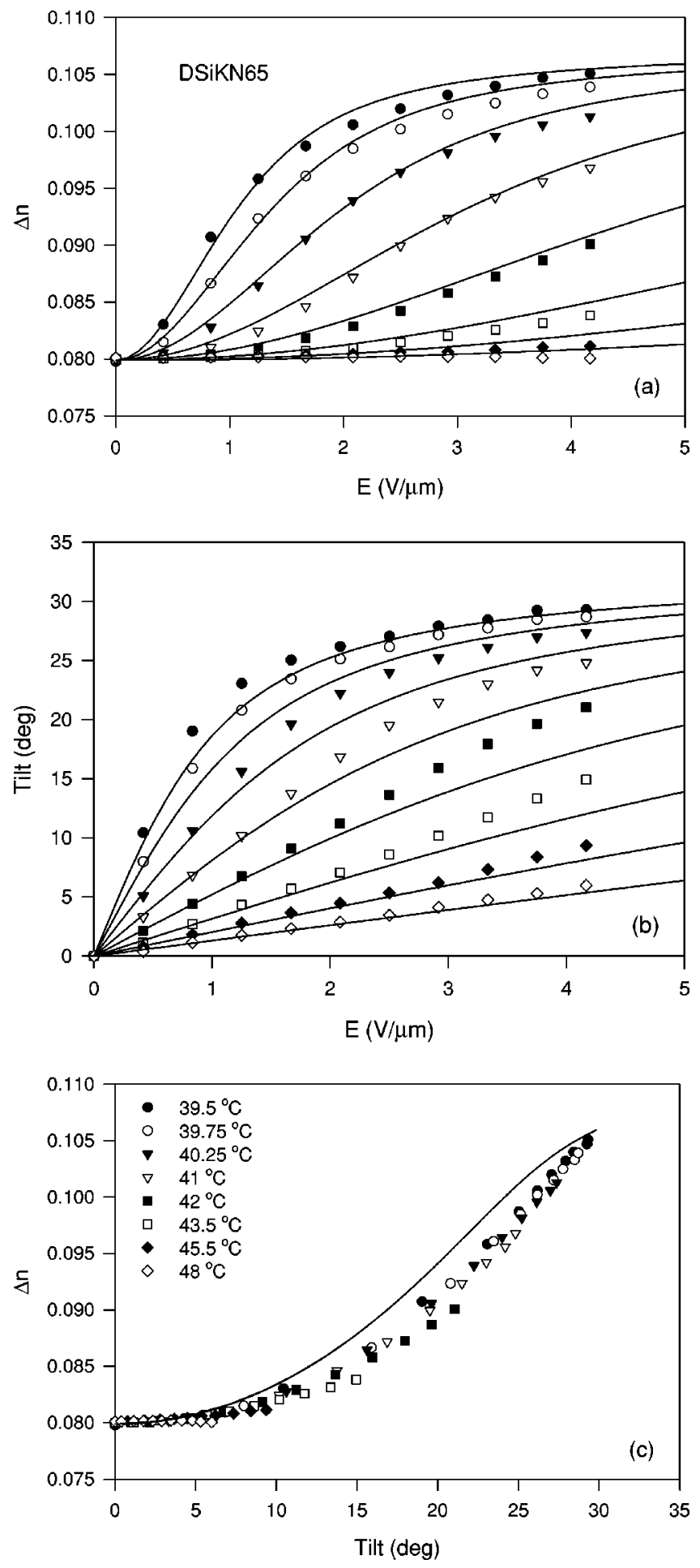

FIG. 2. Symbols, data for the field-dependent birefringence and tilt of DSiKN65 at several temperatures: 39.5, 39.75, 40.25, 41, 42, 43.5, 45.5, and $48^{\circ} \mathrm{C}$ (top to bottom). Lines, fits for the fielddependent birefringence and tilt at the same temperatures (top to bottom). (a) Birefringence vs field. (b) Tilt vs field. (c) Birefringence vs tilt.

field-dependent birefringence in the siloxane-containing compounds.

In this paper, we go beyond that preliminary communication to present a detailed theoretical and experimental study 

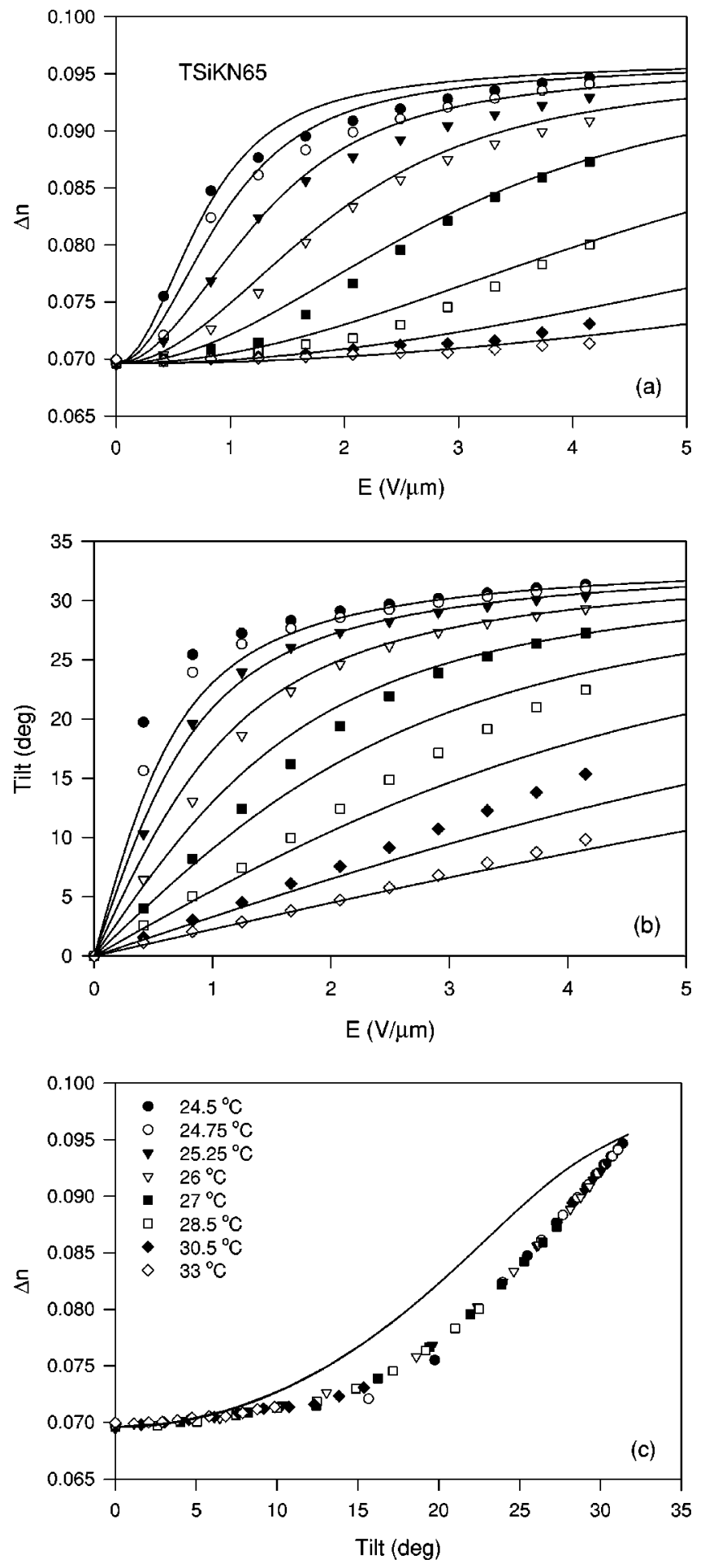

FIG. 3. Symbols, data for the field-dependent birefringence and tilt of TSiKN65 at several temperatures: 24.5, 24.75, 25.25, 26, 27 , $28.5,30.5$, and $33^{\circ} \mathrm{C}$ (top to bottom). Lines, fits for the fielddependent birefringence and tilt at the same temperatures (top to bottom). (a) Birefringence vs field. (b) Tilt vs field. (c) Birefringence vs tilt.

of the optical tilt and birefringence in two of the siloxanecontaining liquid crystals, DSiKN65 and TSiKN65. On the experimental side, we measure the tilt and birefringence as functions of temperature as well as applied electric field.
These measurements show that the tilt and birefringence depend sensitively on temperature near the Sm- $A-\mathrm{Sm}-C$ phase transition. On the theoretical side, we develop a systematic model for the orientational distribution in de Vries-type materials through a series of manipulations of the dielectric tensor, and we note that this model predicts the optical tilt as well as the birefringence. Hence, we use the model to fit the ensemble of data for tilt and birefringence as functions of field and temperature. The overall quality of the fits is fairly good, considering that a simple model is being applied to a large data set. For that reason, we can conclude that the model captures the essential features of the orientational ordering in de Vries-type materials. Furthermore, comparison between theory and experiment allows us to extract an important theoretical parameter, the effective dipole moment, as a function of temperature. We find that this quantity scales as a power law near the $\mathrm{Sm}-A-\mathrm{Sm}-C$ transition. The scaling is consistent with predictions from the theory of critical phenomena.

The outline of this paper is as follows. In Sec. II we present the experimental method and results, showing the dependence of optical tilt and birefringence on both the electric field and temperature. In Sec. III we develop the theory for the orientational ordering in de Vries-type materials, leading to predictions for optical tilt and birefringence. We compare the theory with the experiment in Sec. IV, in order to assess the quality of the fit and extract the effective dipole moment. In Sec. V we discuss the results and present the overall conclusions of this theoretical and experimental work.

\section{EXPERIMENT}

The two siloxane-containing compounds used in this investigation, DSiKN65 and TSiKN65, have the structure shown in Fig. 1. DSiKN65 has a Sm- $C-\mathrm{Sm}-A$ transition at $39^{\circ} \mathrm{C}$ and a Sm- $A$-isotropic transition at $55^{\circ} \mathrm{C}$, while TSiKN65 has these transitions at $24{ }^{\circ} \mathrm{C}$ and $56^{\circ} \mathrm{C}$, respectively. These liquid crystals were vacuum filled into EHC cells of $5 \mu \mathrm{m}$ thickness with rubbed polyimide surfaces. The bookshelf geometry of the Sm- $A$ phase was achieved by extremely slow cooling through the isotropic-Sm- $A$ transition in the presence of a $1 \mathrm{~Hz}$ bipolar square-wave electric field with an amplitude of $5 \mathrm{~V} / \mu \mathrm{m}$. The temperature of the sample cell was regulated by an Instec mK-2 controller and HS-1 hotstage. The temperature gradient across the portion of the sample being illuminated was less than $0.1 \mathrm{~K}$. The hotstage was placed on the rotable stage of a polarizing microscope with a $10 \times$ eyepiece and $5 \times$ objective. The light from a halogen lamp passed through a $633 \mathrm{~nm}$ filter (fullwidth at half maximum of $3 \mathrm{~nm}$ ) before encountering the sample. The intensity of the transmitted light was measured by a silicon diode detector, amplifier, and oscilloscope. At each temperature, various electric-field values were applied to the sample by a bipolar $10 \mathrm{~Hz}$ square wave.

For a homogeneous liquid crystal sample between crossed polarizers, with its director perpendicular to the light propagation direction, the transmitted intensity $I_{\perp}(\gamma)$ is given by 


$$
I_{\perp}(\gamma)=I_{\min }+I_{0} \sin ^{2}(\delta / 2) \sin ^{2}(2 \gamma),
$$

where $I_{\min }$ is the background intensity, $I_{0}$ is the incident intensity, $\gamma$ is the angle between the director and either of the polarizer axes, and $\delta$ is the phase retardation angle. The latter angle depends on the birefringence $\Delta n$, the sample thickness $d$, and the wavelength of light $\lambda$ through

$$
\delta=2 \pi d \Delta n / \lambda
$$

As the sample stage is rotated, the maximum value $\left[I_{\perp}(\gamma)\right]_{\max }$ occurs when $\gamma=\pi / 4$, and the minimum value $I_{\text {min }}$ occurs when $\gamma=0$.

If the polarizers are parallel to each other instead of being crossed, the transmitted light intensity $I_{\|}(\gamma)$ is

$$
I_{\|}(\gamma)=I_{\min }+I_{0}\left[1-\sin ^{2}(\delta / 2) \sin ^{2}(2 \gamma)\right]
$$

Rotation of the sample stage yields the maximum value, $\left[I_{\|}(\gamma)\right]_{\max }=I_{\min }+I_{0}$ at $\gamma=0$, and the minimum value $\left[I_{\|}(\gamma)\right]_{\min }$ when $\gamma=\pi / 4$. Measurement of the minimum and maximum values of the intensity with the two polarizer configurations in place can be used to find the phase retardation angle

$$
\delta=2 \sin ^{-1} \sqrt{\frac{\left[I_{\perp}(\gamma)\right]_{\max }-I_{\min }}{\left[I_{\|}(\gamma)\right]_{\max }-I_{\min }}}
$$

and hence the birefringence $\Delta n$.

The tilt angle can be easily measured by rotating the sample stage so that $I_{\perp}(\gamma)$, the transmitted intensity with the polarizers crossed, is equal for both halves of the bipolar square wave. In the two halves of the square wave, the director orientation is $\gamma=\gamma_{0} \pm \theta_{\text {tilt }}$, where $\gamma_{0}$ is the orientation of the layer normal relative to either polarizer axis and $\theta_{\text {tilt }}$ is the electroclinic tilt angle. If the intensities are equal, then $\gamma_{0}=0$ and hence the intensity $\left[I_{\perp}\right]_{ \pm}$is just

$$
\left[I_{\perp}\right]_{ \pm}=I_{\min }+I_{0} \sin ^{2}(\delta / 2) \sin ^{2}\left(2 \theta_{\text {tilt }}\right) .
$$

If this measurement is combined with the measurements of the maximum and minimum intensities with crossed polarizers, the tilt angle can be determined as

$$
\theta_{\text {tilt }}=\frac{1}{2} \sin ^{-1} \sqrt{\frac{\left[I_{\perp}\right]_{ \pm}-I_{\min }}{\left[I_{\perp}(\gamma)\right]_{\max }-I_{\min }}} .
$$

We measured the tilt angle and birefringence for eleven values of the electric field at eight values of the temperature, starting just above the Sm-C-Sm- $A$ transition and ending roughly $10 \mathrm{~K}$ above the transition. The data for DSiKN65 are shown by the symbols in Figs. 2(a) and 2(b), and the data for TSiKN65 are shown in Figs. 3(a) and 3(b). Several features of the data are clear from these figures. The tilt angle increases linearly with electric field at low field and then saturates at an asymptotic value at high field. The birefringence increases quadratically with field at low field and then saturates. Both of these quantities depend more sensitively on field near the Sm- $C-\mathrm{Sm}-A$ transition temperature than at higher temperature, away from the transition. By comparison with "conventional" electroclinic liquid crystals that follow the rigid-rod scenario, such as KN125 [20], the birefringence of these materials is much smaller and varies much more with electric field.

For an alternative way to look at the data, we plot the birefringence vs tilt angle for DSiKN65 and TSiKN65 in Figs. 2(c) and 3(c), respectively. The most striking feature of these plots is that, for each material, the measurements at all temperatures collapse onto a single universal curve. The shape of this curve is approximately a parabola.

\section{THEORY}

To explain the dependence of the birefringence and tilt of DSiKN65 and TSiKN65 on electric field and temperature, we develop a theory for orientational ordering in de Vriestype materials. This theory is related to the theory for fieldinduced biaxiality in "conventional" rigid-rod $\mathrm{Sm}-A$ liquid crystals [20]. It is also similar to the "random model" for the optical properties of $\mathrm{V}$-shaped switching materials [22,23]. One difference from the latter is that it takes into account the inherent biaxiality of the molecules.

This theory is based on a rotational averaging of the dielectric tensor $\epsilon$. In the coordinate system of a single molecule, the dielectric tensor at optical frequencies has the diagonal form

$$
\boldsymbol{\epsilon}=\left(\begin{array}{ccc}
\epsilon_{a} & 0 & 0 \\
0 & \epsilon_{b} & 0 \\
0 & 0 & \epsilon_{c}
\end{array}\right),
$$

where $a, b$, and $c$ are the principal dielectric axes of the molecule. Let the $c$ axis represent the long axis of the molecule, while $a$ and $b$ are orthogonal to that axis. To transform this tensor into the laboratory coordinate system, we make two rotations. First, to represent the tilt of the molecule with respect to the smectic layer normal, we rotate through the polar angle $\eta$ about the molecular $b$ axis. Second, to represent the orientation of the tilt direction in the smectic layer plane, we rotate through the azimuthal angle $\phi$ about the laboratory $z$ axis, the smectic layer normal. The result of these two rotation operations is 


$$
\epsilon=\left(\begin{array}{ccc}
\epsilon_{a} \cos ^{2} \eta \cos ^{2} \phi & -\epsilon_{b} \cos \phi \sin \phi & \\
+\epsilon_{c} \sin ^{2} \eta \cos ^{2} \phi & +\epsilon_{a} \cos ^{2} \eta \cos \phi \sin \phi & \left(\epsilon_{c}-\epsilon_{a}\right) \cos \eta \sin \eta \cos \phi \\
+\epsilon_{b} \sin ^{2} \phi & +\epsilon_{c} \sin ^{2} \eta \cos \phi \sin \phi & \\
& & \\
-\epsilon_{b} \cos \phi \sin \phi & \epsilon_{a} \cos ^{2} \eta \sin ^{2} \phi & \\
+\epsilon_{a} \cos ^{2} \eta \cos \phi \sin \phi & +\epsilon_{c} \sin ^{2} \eta \sin ^{2} \phi & \left(\epsilon_{c}-\epsilon_{a}\right) \cos \eta \sin \eta \sin \phi \\
+\epsilon_{c} \sin ^{2} \eta \cos \phi \sin \phi & +\epsilon_{b} \cos ^{2} \phi & \\
& & \\
\left(\epsilon_{c}-\epsilon_{a}\right) \cos \eta \sin \eta \cos \phi & \left(\epsilon_{c}-\epsilon_{a}\right) \cos \eta \sin \eta \sin \phi & \epsilon_{a} \sin ^{2} \eta+\epsilon_{c} \cos ^{2} \eta
\end{array}\right) .
$$

We now make three assumptions about the distribution of molecular orientations. First, we suppose that all molecules have the same value of the polar angle $\eta$, which characterizes the tilt cone. For simplicity, we suppose this angle is independent of the temperature and applied field. Second, we suppose that the molecules have a distribution of the azimuthal angle $\phi$. In zero field this distribution is uniform, but under an applied electric field $E$ (in the $y$ direction) this distribution must be biased (in favor of tilt in the $x$ direction). We assume the mean-field distribution function

$$
\rho(\phi)=\rho_{0} \exp \left(E P_{0} \cos \phi / k_{B} T\right),
$$

where $\rho_{0}$ is a normalization factor, $T$ is the temperature, and $P_{0}$ is an effective dipole moment coupling to the electric field, which will be discussed further below. Third, we suppose that there is no distribution of rotations about the molecular long axes, i.e., all the molecules have a unique value of the third Euler angle. This simplifying assumption is justified by the idea that whatever microscopic interaction favors molecular tilt must prefer a particular part of the molecule to point down toward the smectic layers. It implies that the molecular dipole moments are in the smectic layer plane, tangent to the tilt cone.

Given these assumptions, we can average the dielectric tensor (8) over the distribution function (9). The result is

$$
\epsilon=\left(\begin{array}{ccc}
\epsilon_{a} \cos ^{2} \eta\left(\frac{1}{2}+\frac{1}{2} \frac{I_{2}\left(E P_{0} / k_{B} T\right)}{I_{0}\left(E P_{0} / k_{B} T\right)}\right) & 0 \\
+\epsilon_{c} \sin ^{2} \eta\left(\frac{1}{2}+\frac{1}{2} \frac{I_{2}\left(E P_{0} / k_{B} T\right)}{I_{0}\left(E P_{0} / k_{B} T\right)}\right) & \left(\epsilon_{c}-\epsilon_{a}\right) \cos \eta \sin \eta \frac{I_{1}\left(E P_{0} / k_{B} T\right)}{I_{0}\left(E P_{0} / k_{B} T\right)} \\
+\epsilon_{b}\left(\frac{1}{2}-\frac{1}{2} \frac{I_{2}\left(E P_{0} / k_{B} T\right)}{I_{0}\left(E P_{0} / k_{B} T\right)}\right) & \epsilon_{a} \cos ^{2} \eta\left(\frac{1}{2}-\frac{1}{2} \frac{I_{2}\left(E P_{0} / k_{B} T\right)}{I_{0}\left(E P_{0} / k_{B} T\right)}\right) & 0 \\
0 & +\epsilon_{c} \sin ^{2} \eta\left(\frac{1}{2}-\frac{1}{2} \frac{I_{2}\left(E P_{0} / k_{B} T\right)}{I_{0}\left(E P_{0} / k_{B} T\right)}\right) & \\
\left(\epsilon_{c}-\epsilon_{a}\right) \cos \eta \sin \eta \frac{I_{1}\left(E P_{0} / k_{B} T\right)}{I_{0}\left(E P_{0} / k_{B} T\right)} & +\epsilon_{b}\left(\frac{1}{2}+\frac{1}{2} \frac{I_{2}\left(E P_{0} / k_{B} T\right)}{I_{0}\left(E P_{0} / k_{B} T\right)}\right) & \epsilon_{a} \sin ^{2} \eta+\epsilon_{c} \cos ^{2} \eta
\end{array}\right)
$$

where $I_{0}, I_{1}$, and $I_{2}$ are the modified Bessel functions.

To model the experimental results, we must predict the optical properties of a sample for light propagating in the $y$ direction, parallel to the applied electric field. For that reason, we diagonalize the average dielectric tensor in the $x z$ plane. The eigenvectors give the principal optical axes of the sample. In particular, the optical tilt $\theta(E)$ is the angle be- tween the eigenvectors and the $x$ and $z$ axes. The eigenvalues give the dielectric constants along the principal optical axes. The indices of refraction are the square roots of these dielectric constants, and the birefringence is then the difference between these square roots.

This diagonalization can be done exactly in the two limiting cases of low field and high field. For $E \rightarrow 0$, the tensor 
is already diagonal, and we obtain

$$
\theta(0)=0 \text {, }
$$

$\Delta n(0)=\sqrt{\epsilon_{a} \sin ^{2} \eta+\epsilon_{c} \cos ^{2} \eta}-\sqrt{\frac{\epsilon_{a} \cos ^{2} \eta+\epsilon_{c} \sin ^{2} \eta+\epsilon_{b}}{2}}$

By comparison, for $E \rightarrow \infty$, diagonalization gives

$$
\begin{gathered}
\theta(\infty)=\eta, \\
\Delta n(\infty)=\sqrt{\epsilon_{c}}-\sqrt{\epsilon_{a} .}
\end{gathered}
$$

Note that the high-field limit shows that maximum possible birefringence, which comes from the difference between the dielectric constant $\epsilon_{c}$ along the long axis of the molecule and the dielectric constant $\epsilon_{a}$ perpendicular to the long axis. The low-field limit shows a lower birefringence, because it mixes the dielectric components in a rotational average.

For intermediate values of the electric field, we diagonalize the tensor numerically using MATHEMATICA. This numerical procedure shows that the predicted birefringence and tilt have the same general form as the experimental data. For low fields, the tilt increases linearly and the birefringence increases quadratically with field. They both saturate around a field of $k_{B} T / P_{0}$ and approach a limiting value at high field. The question is thus how well the prediction can fit the data for birefringence and tilt simultaneously.

Before we go on to the fits, we should briefly discuss the interpretation of the parameter $P_{0}$. In the mean-field distribution function of Eq. (9), $P_{0}$ is the effective dipole moment that couples to the applied electric field. Because the molecules undergo orientational fluctuations in large correlated groups, $P_{0}$ can be much greater than the dipole moment of a single molecule. Near a second-order transition from the Sm- $A$ to the Sm- $C$ phase, it should increase as a power law. Because $P_{0}$ represents the susceptibility of the tilt angle to an applied electric field, it should scale with the susceptibility exponent $\gamma$

$$
P_{0}(T) \propto\left(T-T_{A C}\right)^{-\gamma} .
$$

The Sm- $A-\mathrm{Sm}-C$ transition should be in the universality class of the three-dimensional $x y$ model, and hence we expect $\gamma \approx 1.33$ [24]. This expected scaling will be tested by the fits in the following section.

\section{FITTING}

To compare the theory with the experimental data, we note that the theory involves five parameters: the cone angle $\eta$, the dielectric parameters $\epsilon_{a}, \epsilon_{b}$, and $\epsilon_{c}$, and the effective dipole moment $P_{0}$. The first four of these parameters should be independent of temperature and should depend only on the liquid-crystalline material, while the last parameter $P_{0}$ should be a function of temperature.

To determine the cone angle $\eta$, we use the limiting value of the tilt data at high field, following Eq. (12a). We use the lowest-temperature data set because it has the clearest fea-
TABLE I. Fit parameters for the two liquid crystals studied. The first four parameters are temperature independent, while $P_{0}$ depends on temperature.

\begin{tabular}{lcccc}
\hline \hline Parameter & Temp. $\left({ }^{\circ} \mathrm{C}\right)$ & DSiKN65 & Temp. $\left({ }^{\circ} \mathrm{C}\right)$ & TSiKN65 \\
\hline$\eta$ & & $33^{\circ}$ & & $34^{\circ}$ \\
$\epsilon_{a}$ & 2.484 & & 2.493 \\
$\epsilon_{b}$ & & 2.360 & & 2.379 \\
$\epsilon_{c}$ & & 2.836 & & 2.808 \\
\hline$P_{0}(T)(\mathrm{D})$ & 39.5 & 1768 & 24.5 & 2390 \\
& 39.75 & 1373 & 24.75 & 1952 \\
& 40.25 & 946 & 25.25 & 1420 \\
& 41.0 & 614 & 26.0 & 961 \\
& 42.0 & 386 & 27.0 & 633 \\
& 43.5 & 233 & 28.5 & 376 \\
& 45.5 & 150 & 30.5 & 224 \\
& 48.0 & 97 & 33.0 & 154 \\
\hline \hline
\end{tabular}

tures. To determine the dielectric parameters $\epsilon_{a}, \epsilon_{b}$, and $\epsilon_{c}$, we use the limiting values of the birefringence data at low and high fields, again using the lowest-temperature data set. Equations (11b) and (12b) then give two constraints on the three dielectric parameters. For a third constraint, we assume that the isotropically averaged index of refraction $\sqrt{\left(\epsilon_{a}+\epsilon_{b}+\epsilon_{c}\right) / 3}=1.6$. This value of 1.6 is just a typical value for an organic liquid, and we have confirmed that the results are not sensitive to this particular choice. With these three constraints, we can solve for $\epsilon_{a}, \epsilon_{b}$, and $\epsilon_{c}$. The results for all the temperature-independent parameters are listed in Table I. Note that the cone angles are very similar, $33^{\circ}$ in DSiKN65 and $34^{\circ}$ in TSiKN65, and the dielectric parameters are also quite similar between the liquid crystals. Presumably this is due to the chemical similarity between these two materials.

Once those parameters are determined, there is only one remaining temperature-dependent fitting parameter $P_{0}(T)$. To determine this parameter, we fit the combined data for tilt vs field and birefringence vs field at each temperature. In this fit, we must combine the two contributions to $\chi^{2}$ with appropriate weighting factors. A reasonable choice is to weight the birefringence data (unitless) by a factor of 1000 relative to the tilt data (in radians), which gives equally good fits to both data sets. The fits are shown by the solid lines in Figs. 2(a) and (b) and 3(a) and (b), and the extracted values of $P_{0}(T)$ are listed in Table I. Clearly the theory captures the field dependence of the tilt and birefringence data. The fits are qualitatively good for all of the data and quantitatively good for most of the data.

An alternative way to look at the data is to plot the birefringence vs tilt angle. As mentioned in Sec. II, the data at all temperatures collapse onto a single universal curve for each material. This data collapse is indeed a feature of the theory: Because the average dielectric tensor of Eq. (10) depends on field and temperature only through the combination $E P_{0}(T) / k_{B} T$, the theory predicts a universal curve that depends only on $\eta, \epsilon_{a}, \epsilon_{b}$, and $\epsilon_{c}$. In Figs. 2(c) and 3(c), we plot the theoretical curve along with the data. Note that the 
end points of this curve are fixed by the fit parameters, but the shape of the curve between the end points is determined by the theory with no further choice of parameters. This shape is generally close to the data, although there is some clear discrepancy.

We have tried slightly different estimates for the zero-field and high-field limits of the tilt and birefringence, as well as a different fitting procedure that determines all the parameters from the birefringence data and then uses them to calculate the tilt angle. The results of all these variations are quite similar to what is shown here. The differences between the theoretical curves and the data are always present at about the same level.

Note that these fits imply that the molecules are biaxial, with $\epsilon_{a} \neq \epsilon_{b} \neq \epsilon_{c}$. For comparison, we considered a uniaxial model with $\epsilon_{a}=\epsilon_{b} \neq \epsilon_{c}$. This model gives good fits to the birefringence data, but it implies a cone angle $\eta$ of $24^{\circ}-26^{\circ}$, which is less than the observed tilt angle. As a result, the fits involving the tilt angle ( $\theta$ vs $E$ and $\Delta n$ vs $\theta$ ) are unsatisfactory. (This inconsistency occurs even if we eliminate the constraint on the isotropically averaged index of refraction.)

In Sec. III, we argued that the value of $P_{0}(T)$ should increase as the temperature decreases toward the Sm- $A-\mathrm{Sm}-C$ transition. The fit results in Table I are consistent with this trend. To analyze the temperature dependence, we plot $P_{0}$ vs $T$ in Figs. 4(a) and (b) and fit the data to the power law

$$
P_{0}(T)=A\left(\frac{T-T_{A C}}{T_{A C}}\right)^{-\gamma}
$$

The power law gives a very good fit to the observed temperature dependence, with the fitting parameters listed in Table II. Note that the exponent $\gamma$ is 1.51 for DSiKN65 and 1.75 for TSiKN65. This exponent is somewhat larger than the expected value of 1.33 , but we do not have enough data close to the transition to determine the exponent precisely. Overall, the fitting results are consistent with the theoretical concept that $P_{0}$ is an effective dipole moment that grows larger as the system approaches the Sm- $A-\mathrm{Sm}-C$ transition, following a power-law scaling relation.

A further consistency check comes from the amplitude of the power-law variation. The amplitude $A$ is $0.54 \mathrm{D}$ in DSiKN65 and $0.44 \mathrm{D}$ in TSiKN65, where 1D $=10^{-18}$ esu $\mathrm{cm}$. This is the same order of magnitude as a typical molecular dipole moment of 1-2 D [25]. Over the experimental temperature range, $P_{0}(T)$ increases from roughly $10^{2}$ to $10^{3}$ times this value.

One aspect of the fitting results for $P_{0}(T)$ is surprising. Experimentally, the $\mathrm{Sm}-A-\mathrm{Sm}-C$ transition occurs within $0.5^{\circ} \mathrm{C}$ of the lowest temperature for which tilt angle and birefringence were measured. However, the fits for $P_{0}(T)$ shown in Table II indicate a second-order transition temperature almost $2{ }^{\circ} \mathrm{C}$ below the actual transition temperature. Power-law fits to the tilt angle and birefringence data vs temperature at the lowest nonzero value of the electric field also indicate second-order transition temperatures consistent with those in Table II.
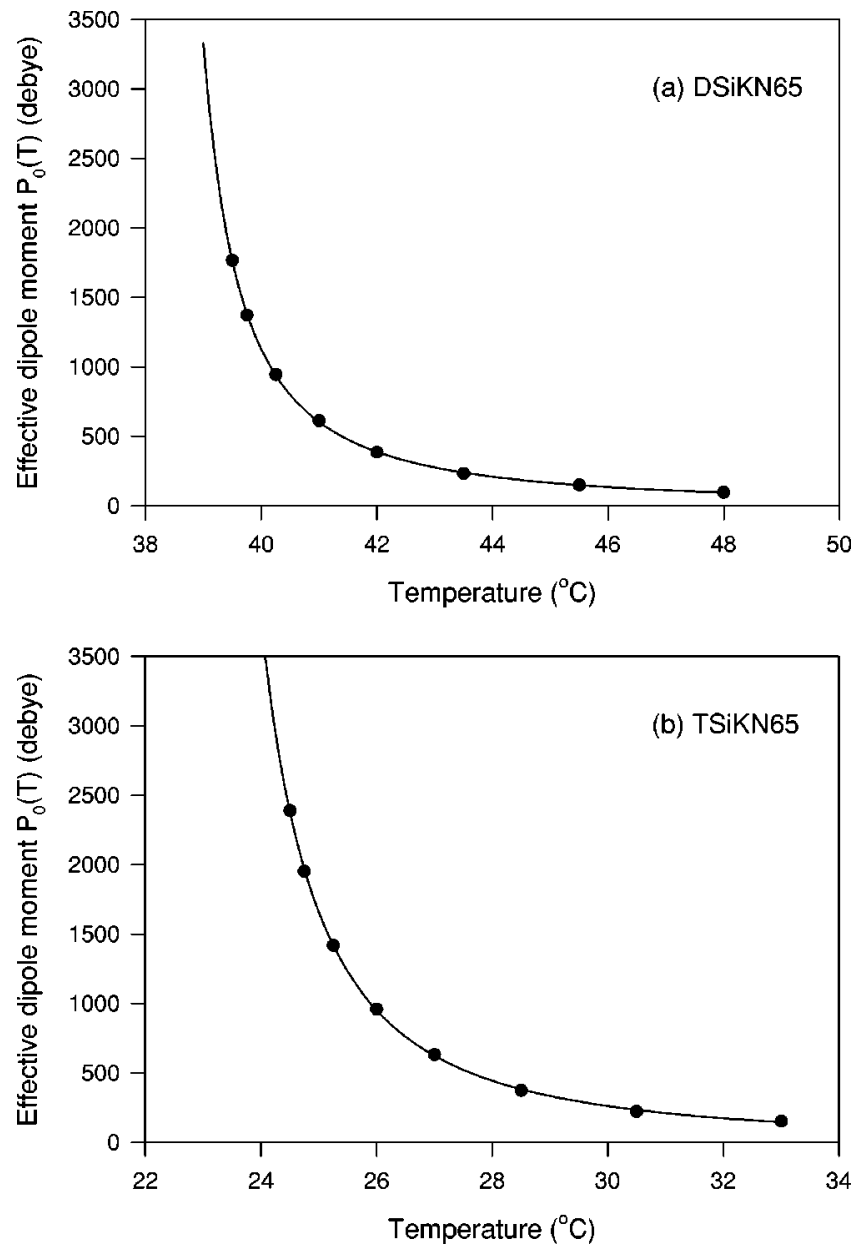

FIG. 4. Symbols, effective dipole moment $P_{0}(T)$, from Table I. Lines, power-law fits for the temperature dependence of $P_{0}(T)$. (a) DSiKN65. (b) TSiKN65.

One possible explanation for the difference between the experimental and the fit transition temperatures is that the transition is weakly first order, with a small discontinuous change in the tilt angle and birefringence. To test this possibility, we looked for hysteresis upon heating and cooling through the transition in DSiKN65 using a differential scanning calorimeter. The transition always occurred at a higher temperature upon heating as opposed to cooling. When this temperature difference was plotted vs the heating/cooling rate $\left(0.02-0.30^{\circ} \mathrm{C} / \mathrm{min}\right)$, it extrapolated linearly to $0.05^{\circ} \mathrm{C}$ at zero heating/cooling rate. This hysteresis indicates that the transition has a slight first-order character.

Another possible explanation for this difference is that there is another phase between the Sm- $C$ and $\mathrm{Sm}-A$ phases.

TABLE II. Power-law fit parameters for the temperature dependence of the $P_{0}(T)$ data in Table I.

\begin{tabular}{lcc}
\hline \hline Parameter & DSiKN65 & TSiKN65 \\
\hline$\gamma$ & $1.51 \pm 0.06$ & $1.75 \pm 0.08$ \\
$T_{A C}\left({ }^{\circ} \mathrm{C}\right)$ & $38.0 \pm 0.1$ & $22.3 \pm 0.1$ \\
$A(\mathrm{D})$ & $0.54 \pm 0.13$ & $0.44 \pm 0.12$ \\
\hline \hline
\end{tabular}


Since this transition involves the establishment of long-range azimuthal order of the tilt, there could be an intermediate phase, perhaps one with a discrete distribution of azimuthal angles. We see no evidence for this in the optical and differential scanning calorimetry data, but these types of measurements may be insensitive to such structural changes.

\section{DISCUSSION}

In this paper, we have presented a theory for the orientational distribution of molecules in de Vries-type Sm- $A$ liquid crystals. This theory makes the simplest possible assumptions about the distribution of molecular orientations on a tilt cone, and gives predictions for the dependence of tilt angle and birefringence on electric field and temperature. We have compared these predictions with experimental data for the tilt and birefringence near the Sm- $A-\mathrm{Sm}-C$ transition in the two materials DSiKN65 and TSiKN65. The overall quality of the fits is good, considering that we are fitting a simple model to a large amount of data over a wide range of electric field and reduced temperature. Furthermore, the fits give quantitatively reasonable values for the effective dipole moment, and show how this quantity increases as the system approaches the $\mathrm{Sm}-A-\mathrm{Sm}-C$ transition.

While the agreement between the theory and the experiment is generally good, there are clearly some deviations. These deviations show that the experimental system has some behavior that is more complex than the simple assumptions of the theory. First, the cone angle probably has some dependence on temperature and electric field. This dependence is shown by measurements of the layer spacing in TSiKN65 [19]: For the range of electric field and temperature that we have studied, the layer spacing changes by roughly $0.6 \%$ with field and roughly $0.1 \%$ with temperature. Second, the molecules may have a distribution of rotations about the molecular long axes, i.e. a distribution of dipole moment orientations relative to the tilt cone, and this distribution may change as a function of field and temperature. Third, the system may have a distribution of molecular con- formations, and this distribution may also change with field and temperature. We have not considered these effects in our current theory, because we wish to explain the main trends in the data with the simplest possible theory and to avoid adding further fitting parameters. However, these effects can be studied in future work.

As a final point, we speculate that there are not really two separate classes of $\mathrm{Sm}-A$ liquid crystals: "conventional" and de Vries type. Rather, there may be a whole spectrum of materials between these two extremes. On one end of the spectrum are $\mathrm{Sm}-A$ liquid crystals with a very small cone angle. When an electric field is applied, the main response is that the molecules tilt uniformly by much more than the cone angle. These are the "conventional" Sm- $A$ materials that undergo layer contraction. On the other end of the spectrum are $\mathrm{Sm}-A$ liquid crystals with large cone angles. As an electric field is applied, the main response is the establishment of long-range azimuthal order, with a relatively small change in the magnitude of the cone angle. These are the Sm- $A$ materials that tilt with extremely little layer contraction. In between these limiting cases, other liquid crystals may undergo substantial changes in both the cone angle and the azimuthal distribution in response to an electric field. The materials that we have studied, DSiKN65 and TSiKN65, are clearly near the de Vries limit of this spectrum, but their response to an electric field gives insight into the full range of behavior that is possible in the $\mathrm{Sm}-A$ phase.

\section{ACKNOWLEDGMENTS}

We would like to thank J. Naciri for synthesizing the liquid crystals, B. R. Ratna and M. S. Spector for helpful discussions, and B. T. Weslowski for assistance with the experiments. We particularly thank F. J. Bartoli, S. R. Flom, and J. $\mathrm{R}$. Lindle for their collaboration on an earlier part of this study, published in Ref. [21]. This research was supported by the Office of Naval Research and the Naval Research Laboratory.
[1] R. B. Meyer, Mol. Cryst. Liq. Cryst. 40, 33 (1977).

[2] S. Garoff and R. B. Meyer, Phys. Rev. Lett. 38, 848 (1977).

[3] N. Collings, W. A. Crossland, R. C. Chittick, and M. F. Bone, Proc. SPIE 963, 46 (1989).

[4] G. Andersson, I. Dahl, L. Komitov, S. T. Lagerwall, K. Skarp, and B. Stebler, J. Appl. Phys. 66, 4983 (1989).

[5] P. A. Williams, N. A. Clark, M. B. Ros, D. M. Walba, and M. D. Wand, Ferroelectrics 121, 143 (1991).

[6] B. R. Ratna, G. P. Crawford, S. K. Prasad, J. Naciri, P. Keller, and R. Shashidhar, Ferroelectrics 148, 425 (1993).

[7] T. Carlsson, B. Žekš, C. Filipič, A. Levstik, and R. Blinc, Mol. Cryst. Liq. Cryst. 163, 11 (1988); B. Žekš and R. Blinc, in Ferroelectric Liquid Crystals (Gordon and Breach, Philadelphia, 1991), p. 365.

[8] I. Abdulhalim and G. Moddel, Liq. Cryst. 9, 493 (1991).

[9] A. de Vries, A. Ekachai, and N. Spielberg, Mol. Cryst. Liq. Cryst. Lett. 49, 143 (1979); A. de Vries, ibid. 49, 179 (1979).
[10] J. Xu, R. L. B. Selinger, J. V. Selinger, B. R. Ratna, and R. Shashidhar, Phys. Rev. E 60, 5584 (1999).

[11] G. P. Crawford, R. E. Geer, J. Naciri, R. Shashidhar, and B. R. Ratna, Appl. Phys. Lett. 65, 2937 (1994).

[12] J. Pavel and M. Glogarova, Ferroelectrics 114, 131 (1991).

[13] K. Skarp, G. Andersson, T. Hirai, A. Yoshizawa, K. Hiraoka, H. Takezoe, and A. Fukuda, Jpn. J. Appl. Phys., Part 1 31, 1409 (1992).

[14] R. E. Geer, S. J. Singer, J. V. Selinger, B. R. Ratna, and R. Shashidhar, Phys. Rev. E 57, 3059 (1998).

[15] F. J. Bartoli, J. R. Lindle, S. R. Flom, R. Shashidhar, G. Rubin, J. V. Selinger, and B. R. Ratna, Phys. Rev. E 58, 5990 (1998).

[16] M. D. Radcliffe, M. L. Brostrom, K. A. Epstein, A. G. Rappaport, B. N. Thomas, R. Shao, and N. A. Clark, Liq. Cryst. 26, 789 (1999).

[17] F. Giesselmann, P. Zugenmaier, I. Dierking, S. T. Lagerwall, B. Stebler, M. Kaspar, V. Hamplova, and M. Glogarova, Phys. Rev. E 60, 598 (1999). 
[18] J. Naciri, J. Ruth, G. Crawford, R. Shashidhar, and B. R. Ratna, Chem. Mater. 7, 1397 (1995).

[19] M. S. Spector, P. A. Heiney, J. Naciri, B. T. Weslowski, D. B. Holt, and R. Shashidhar, Phys. Rev. E 61, 1579 (2000).

[20] F. J. Bartoli, J. R. Lindle, S. R. Flom, J. V. Selinger, B. R. Ratna, and R. Shashidhar, Phys. Rev. E 55, R1271 (1997).

[21] J. R. Lindle, F. J. Bartoli, S. R. Flom, J. V. Selinger, R. Shashidhar, and B. R. Ratna, in Liquid Crystal Materials and Devices, edited by T. J. Bunning et al., MRS Symposia Proceedings No. 559 (Materials Research Society, Pittsburgh, 2000), p. 33.

[22] S. Inui, N. Iimura, T. Suzuki, H. Iwane, K. Miyachi, Y. Takan- ishi, and A. Fukuda, J. Mater. Chem. 6, 671 (1996).

[23] B. Park, S.-S. Seomun, M. Nakata, M. Takahashi, Y. Takanishi, K. Ishikawa, and H. Takezoe, Jpn. J. Appl. Phys., Part 1 38, 1474 (1999).

[24] P. G. de Gennes and J. Prost, The Physics of Liquid Crystals, 2nd ed. (Clarendon Press, Oxford, 1993), Sec. 10.2.1.

[25] J. W. Goodby, R. Blinc, N. A. Clark, S. T. Lagerwall, M. A. Osipov, S. A. Pikin, T. Sakurai, K. Yoshino, and B. Žekš, Ferroelectric Liquid Crystals: Principles, Properties and Applications (Gordon and Breach, Amsterdam, 1991), pp. 12, 288. 